\title{
DISTRIBUCIÓN Y LÍMITES DE LAS ECORREGIONES DEL CENTRO NORTE DE LA PROVINCIA DE SANTA FE SOBRE LA BASE DE LA PRESENCIA DE TIPOS DE BOSQUES
}

\author{
Pensiero, J. F. ${ }^{1}$ \& Bortoluzzi, A. L. ${ }^{1}$
}

\begin{abstract}
RESUMEN
Se proponen los límites geográficos de las ecorregiones de Chaco Húmedo, Chaco Seco y del Espinal para el centro norte de la provincia de Santa Fe. La delimitación se basó ubicaciones de las principales tipos boscosos: algarrobales, quebrachales, quebrachales de tres quebrachos y bosques mixtos, derivadas de relevamientos de campo con GPS a ydatos del mapa de Ordenamiento Territorial de Bosques Nativos. La ubicación de los bosques fue vinculada a celdas hexagonales de 2,5 km de largo y ancho dentro de un grillado que cubrió toda el área analizada. Con las celdas que marcaron presencia de cada clase de bosque se estimó su área de distribución aplicando el algoritmo de envolvente convexa, Los límites se propusieron interpretando la distribución de las clases de bosques junto con información de campo y del análisis florístico. La información generada orientará trabajos de campo para obtener mayores detalles de la vegetación en las áreas limítrofes.
\end{abstract}

Palabras clave: Ecorregiones, biodiversidad, Bosques Nativos.

1.-1.- Facultad de Ciencias Agrarias, Universidad Nacional del Litoral. Kreder 2805. 3080HOF, Esperanza, Santa Fe, Argentina. Email: albortoluzzi@fca.unl.edu.ar

Manuscrito recibido el 13 de abril de 2020 y aceptado para su publicación el 23 de octubre de 2020.

Pensiero J F, Bortoluzzi A L. Distribución y límites de las ecorregiones del centro norte de la provincia de santa fe sobre la base de la presencia de tipos de bosques. FAVE - Ciencias Agrarias 20 (1): 83-98. CC BY-NC-SA 4.0 


\begin{abstract}
Distribution and limits of the ecoregions of the north-central province of Santa Fe based on the presence of forest types.

The geographical limits of the Chaco Húmedo, Chaco Seco and Espinal ecoregions are proposed for the north-central province of Santa Fe. The delimitation was based on locations of the main different forest-type formations: algarrobales, quebrachales, quebrachales of three quebrachos and mixed forests derived from GPS surveys and to locations derived from the Map of Territorial Planning of Native Forests. The location of the forests was linked to hexagonal cells $2.5 \mathrm{~km}$ long and wide within a grid that covered the entire analyzed area. With the cells that marked the presence of each forest class their distribution area was estimated by applying the convex envelope algorithm. The boundaries were proposed interpreting the distribution of forest classes along with field information and floristic analysis. The information generated will orient fieldwork to obtain more details of the vegetation in the bordering areas.
\end{abstract}

Key words: Ecoregions, biodiversity, Native Forest.

\section{INTRODUCCIÓN}

Sobre la base de la flora y vegetación presente, distintos autores han delimitado y clasificado grandes unidades que fueron nominadas, según el caso, como provincias fitogeográficas, provincias biogeográficas, ecorregiones o unidades de vegetación (Cabrera, 1976; Burkart et al., 1999; Brown et al., 2006; Morello et al., 2012; Oyarzabal et al., 2018).

En la provincia de Santa Fe están representadas 5 ecorregiones: Pampa (en el sur), Espinal (centro), Chaco Húmedo (gran parte del centro-norte), Chaco Seco (extremo noroeste) y Deltas e Islas del Paraná (este) (Burkart et al., 1999; Pensiero et al., 2005; Biasatti, 2016).

El presente trabajo se focaliza en el centro y norte de la provincia de Santa Fe, área que posee un amplio historial de uso y transformación por parte de las actividades humanas: tendido de ferrocarriles, rutas y caminos, intervención de los bosques para extracción de tanino, de madera y produc- ción de carbón, aprovechamiento de pastizales para uso ganadero, urbanizaciones, así como la transformación de vastas extensiones para uso agrícola y ganadero (Morello y Rodriguez, 2009; Zarrilli, 2018). Se excluye de este análisis las ecorregiones Pampa y Deltas e Islas del Paraná, cuyos límites, particularmente esta última, están más claramente definidos (Pensiero et al., 2005). Esta última ecorregión se extiende, en el área analizada, en una angosta y larga faja ubicada al este de la ruta provincial 1 y luego, más al norte, de la ruta nacional 11 hasta el cauce con el Río Paraná.

El área que abarca el presente trabajo posee una vegetación muy variada y en particular distintas clases de bosques (Lewis, 1981). Se hallan presentes "Algarrobales", bosques en los que dominan, en el estrato arbóreo, distintas especies del género Prosopis, las más frecuentes y características son Prosopis alba Griseb. ("algarrobo blanco”) y Prosopis nigra (Griseb.) Hieron. var. nigra ("algarrobo negro"), acompañadas por Achatocarpus praecox Griseb. ("tala 
negro”), Aspidosperma quebracho-blanco Schltdl. ("quebracho blanco"), Celtis pallida Torr. ("tala”), Geoffroea decorticans (Gillies ex Hook. \& Arn.) Burkart (“chañar”), Phytolacca dioica L. (“ombú”), Prosopis affinis Spreng. ("ñandubay”), Vachellia caven (Molina) Seigler \& Ebinger ("aromito"), entre otras. En algunos sitios del centro y centro-norte de la Cuña Boscosa, estos algarrobales pueden estar dominados por Prosopis hassleri Harms var. nigroides Burkart ("algarrobo amarillo”), y más al norte, próximo al límite con la provincia del Chaco, por Prosopis vinalillo Stuck. ("vinalillo"). En el centro de la provincia se pueden hallar algarrobales empobrecidos, en los que en el estrato arbóreo aparece con mayor frecuencia Geoffroea decorticans, constituyendo, cuando llega a ser dominante, bosques casi puros denominados “chañarales” (D’Angelo et al., 1987; Lewis, 1991; Hilgert et al., 2003; Pensiero et al., 2005).

Los "Quebrachales" son bosques en los que domina, en el estrato arbóreo, Schinopsis balansae Engler. ("quebracho colorado chaqueño") y ocurren en suelos muy arcillosos y con drenaje impedido o muy dificultoso. Además de la especie dominante, en el estrato arbóreo se pueden observar como acompañantes: Acanthosyris falcata Griseb. ("saucillo"), Achatocarpus praecox, Aspidosperma quebracho-blanco, Aspidosperma triternatum Rojas Acosta ("quebracho lagunero"), Celtis pallida, Geoffroea decorticans, Gleditsia amorphoides (Griseb.) Taub. (“espina corona”), Libidibia paraguariensis (D. Parodi) G.P. Lewis ("guayacán”), Myrcianthes cisplatensis (Cambess.) O. Berg Phil. ("guayabo”), Prosopis alba, Prosopis nigra var. nigra, Prosopis nigra var. ragonesei Burkart ("algarrobo amarillo”), Ruprechtia laxiflora Meisn. ("viraró”), Sarcomphalus mistol (Griseb.) Hauenschild ("mistol”), Senegalia praecox (Griseb.) Seigler \& Ebinger ("garabato”), Sideroxylon obtusifolium Roem. \& Schult.) T.D. Penn. ("guaraniná”), Tabebuia nodosa (Griseb.) Griseb. (“palo cruz”), Vachellia aroma (Gillies ex Hook. \& Arn.) Seigler \& Ebinger ("tusca”), Vachellia caven, entre otras (Lewis, 1991; Lewis et al., 1997; Barberis et al., 1998; Marino y Pensiero, 2003; Pensiero et al., 2005).

Los "Quebrachales de tres quebrachos" son bosques que se caracterizan por la presencia de Schinopsis lorentzii (Griseb.) Engl. ("quebracho colorado santiagueño"), Schinopsis balansae, Aspidosperma quebracho-blanco, Schinopsis heterophylla Ragonese \& J. Castillo ("quebracho colorado mestizo”, híbrido entre $S$. balansae y $S$. lorentzii), acompañados por Libidibia paraguariensis, Parkinsonia praecox (Ruizz \& Pav. ex Hook.) Hakins ("brea”), Prosopis alba, Ruprechtia laxiflora, Sarcomphalus mistol, Senegalia praecox, Tabebuia nodosa, Trithrinax campestris (Burmeist.) Drude \& Griseb. (“carandá”) y, en forma ocasional y muy rara, por Prosopis kuntzei Harms (“itín”) (Pensiero et al., 2005; Torrella et al., 2011).

Los “Bosques Mixtos”, también denominados bosques fuertes o bosques altos, ocurren en los sitios más elevados del terreno y se encuentran en su mayoría hacia el norte y noreste del área estudiada. Estos bosques son los que en la provincia presentan la mayor diversidad específica ya que ellos son el producto de la conjunción de especies de dos linajes, el chaqueño y el paranaense. La característica de estos bosques es que son varias las especies frecuentes en el estrato arbóreo, sin ser ninguna de ellas dominante. Las especies arbóreas más frecuentes son Acanthosyris falcata, Aspi- 
dosperma quebracho-blanco, Cordia americana (L.) Gottschling \& J.S. Mill. ("guayaibí”), Enterolobium contortisiliquum (Vell.) Morong ("timbó colorado"), Ficus luschnathiana (Miq.) Miq. ("higuerón”), Gleditsia amorphoides ("espina corona”), Handroanthus heptaphyllus (Vell.) Maltos ("lapacho rosado"), Holocalyx balansae Micheli (“alecrín”), Libidibia paraguariensis, Luehea divaricana Mart. ("azota caballos”), Myrsine laetevirens (Me) Arechav. (“canelón”), Phyllostylon rhamnoides (J. Poiss.) Taub. ("palo lanza”), Phytolacca dioica, Pisonia zapallo Griseb. var. guaranitica Toursark. ("zapallo caspi”), Prosopis alba, Prosopis nigra var. nigra, Ruprechtia laxiflora, Schinopsis balansae, Syagrus romanzoffiana (Cham.) Glassman ("pindó"), Terminalia triflora (Griseb.) Lillo ("palo amarillo"), entre otras de ocurrencia menor y numerosos arbustos que componen el estrato arbustivo (Lewis et al., 1994; Pensiero et al., 2005).

Otros tipos de vegetación presentes en el área son:

Los Palmares y sabanas, que en la provincia de Santa Fe cubren una importante superficie, siendo los más extensos dominados por Copernicia alba Morong ("caranday”), los que ocurren en suelos bajos, salinos e inundables. Estos palmares pueden presentarse en forma de palmares densos o como extensas sabanas, en este último caso, el estrato herbáceo comúnmente está dominado por un pajonal de Sporobolus spartinus (Trin.) P.M. Peterson \& Saarela ("espartillo"), en suelos salinos, o de Coleataenia prionitis (Nees) Soreng ("paja brava”), en suelos no salinos. Además de las sabanas descriptas, se pueden hallar algunas que ocupan menores superficies, cuyos elementos leñosos corresponden a distintas especies de Prosopis, siendo las más frecuentes Prosopis alba y Prosopis nigra var. nigra, acompañadas por Prosopis affinis y Geoffroea decarticans, y teniendo como dominante en el estrato herbáceo a Elyonurus muticus (Spreng.) Kuntze ("aibe") (Lewis, 1981; D'Angelo et al., 1987; Lewis et al., 1990; Pensiero et al., 2005).

En todo el territorio del área analizada existen distintos tipos de pastizales, con una variada y rica composición florística, los que pueden presentarse en extensos espacios abiertos o formando parte del estrato herbáceo en parques e interior de bosques. Por otra parte, en el área se ubican los Bajos Submeridionales, que constituyen una extensa área de suelos arcillosos, halo-hidromórficos, siendo Natracualf y Natracuol los tipos dominantes, los que pueden permanecer inundados desde fines de verano hasta el invierno, seguidos de una sequía invernal. La vegetación típica de los Bajos se caracteriza por la escasez de elementos arbóreos, siendo los pajonales o espartillares de Sporobolus spartinus la comunidad herbácea que ocupa más de las tres cuartas partes de su superficie. En los sitios más bajos, donde el agua queda retenida por más tiempo, se hallan, aparte de las lagunas, cañadas y esteros cuya vegetación dominante son los canutillares dominados por especies como Echinochloa helodes (Hack.) Parodi ("pasto laguna"), Hymenachne amplexicaulis (Rudge) Nees ("carrizo chico"), Leersia hexandra Sw. ("pastito de agua"), Luziola peruviana Juss. ex J.F. Gmel. ("pastito del agua”), Oplismenopsis najada (Hack. \& Arechav.) Parodi ("canutillo"), entre otras (D’Angelo et al., 1987; Lewis et al., 1990; Stoffela, 1995; Carnevale et al., 1999; Pensiero et al., 2005). 
Dado que las contribuciones de Burkart et al. (1999), Brown y Pacheco (2006) y Morello et al. (2012), presentan divergencias en la delimitación de las ecorregiones del Chaco Húmedo, Chaco Seco y del Espinal para el centro-norte de la provincia de Santa Fe, el presente trabajo propone una nueva delimitación entre dichas ecorregiones considerando la distribución estimada de las clases de bosques y del análisis florístico que caracterizan las mismas.

\section{MATERIALES Y MÉTODOS}

El área de estudio comprendió el centro norte de Santa Fe (Fig. 1), llegando al sur a la Ruta Nacional $N^{\circ} 19$, al oeste y norte los límites con las provincias de Santiago del Estero y Chaco respectivamente y al este con la Ruta Provincial (RP) 1 y su continuación al norte con la Ruta Nacional (RN) 11.

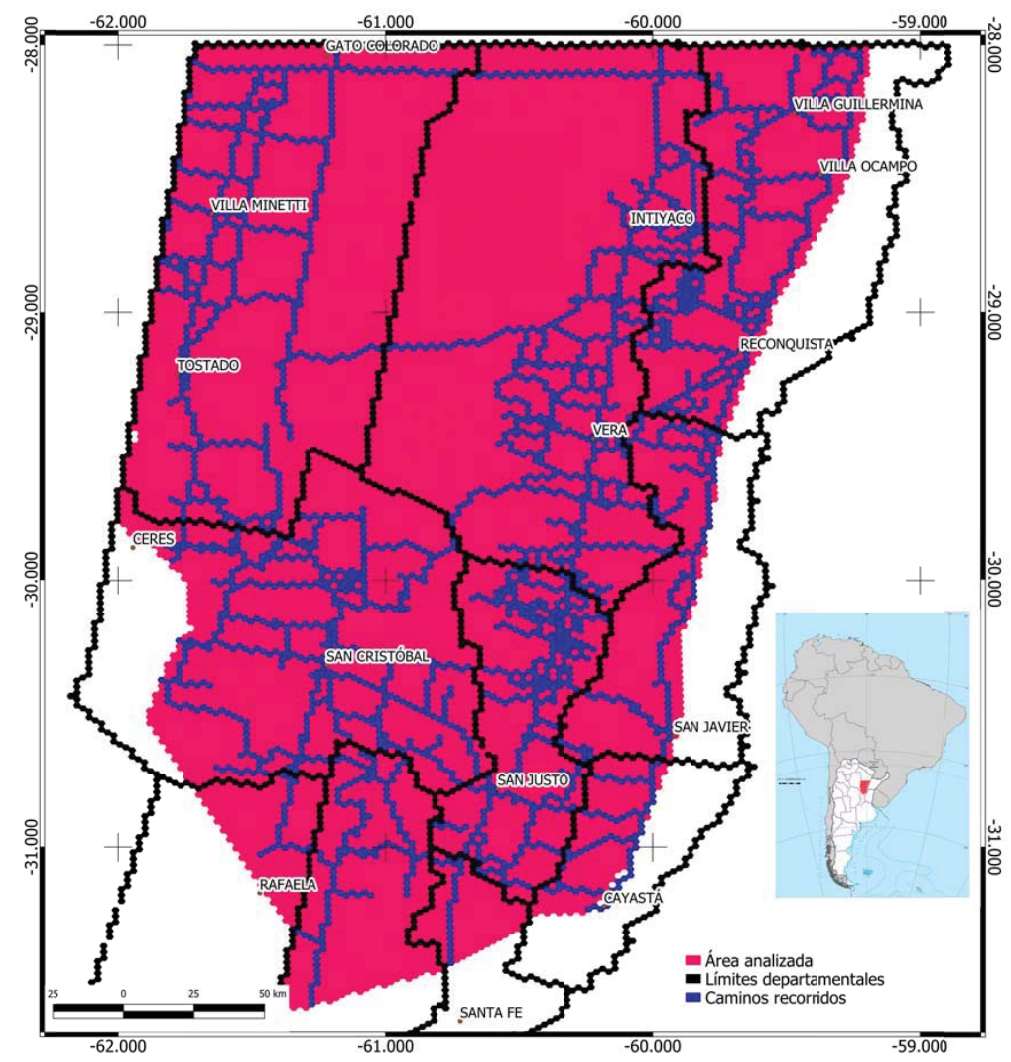

Figura 1. En rojo se indica el área analizada, las líneas azules los caminos recorridos para el relevamiento de datos de campo y las líneas negras los límites departamentales de la provincia de Santa Fe.

Figure 1. The red indicates the analyzed area, the blue lines the paths surveyed and the black lines show the departmental limits of Santa Fe province. 


\section{J. F. Pensiero et al.}

En primer lugar se generó en un Sistema de Información Geográfica, un archivo vectorial de puntos con la ubicación de remanentes de bosques nativos a partir de dos fuentes: A) coordenadas geográficas tomadas mediante el uso de GPS en sucesivas recorridas entre los años 2007 y 2013, por rutas nacionales, provinciales y caminos secundarios de acceso público. En terreno, los datos de GPS fueron asignados a clases de bosques nativos según la similitud con el elenco de especies del estrato arbóreo superior y las acompañantes más relevantes esperable para cada ecorregión señalada por distintos autores (Lewis y Collantes, 1973; Cabrera, 1976; Lewis, 1981; Lewis y Pire, 1981; Burkart et al., 1999; Morello et al., 2012; Pensiero et al., 2005; Oyarzabal et al., 2018). En el caso de bosques intervenidos o en condición degradada, se prestó atención a qué especies correspondían los renovales. B) centroides de los polígonos de clase de bosque nativo del mapa de Ordenamiento Territorial de Bosques Nativos (OTBN) de la provincia de Santa Fe (Ley Provincial 13.372). Este mapa es el resultado de una combinación de clasificaciones semiautomáticas con la interpretación visual de composiciones color de imágenes de los satelitales Landsat 5 y Landsat 7 para el año 2008.

Las ubicaciones de los caminos recorridos y de las clases de bosques del archivo vectorial de puntos fueron vinculadas a un sistema de grilla de celdas hexagonales de 2,5 km de alto y ancho que cubrió toda el área de estudio. De esta forma se convergieron distintas fuentes de información y representación espacial para una misma unidad de análisis. La ventaja de utilizar hexágonos es que permite representar la conectividad de una superficie continua con una relación perímetro/superficie menor a que si se usara celdas cuadradas (Birch et al, 2007). Además, llevar la información a una misma unidad de superficie posibilitó estimar el esfuerzo de muestreo.

Con las celdas que marcaron la presencia de cada clase de bosque (datos de campo más OTBN) se representó su distribución espacial mediante el algoritmo de envolvente convexa (Moreira y Santos, 2007) disponible en el software QGIS (QGIS, 2020). El algoritmo genera un polígono con el área mínima para envolver todos los elementos, en este caso, los centroides de las celdas hexagonales de cada clase de bosque. Para estimar la distribución de la clase Quebrachal, los datos se separaron en dos grupos, uno correspondiente sitios del oeste y otro al este de la provincia.

Los límites de las ecorregiones se propusieron sobre la base de: a) la interpretación de la superposición de las distribuciones de las clases de bosques, complementadas con observaciones de campo; b) el análisis florístico señalado en la bibliografía, sumado a lo registrado a campo y c) los especímenes de herbario (que se conservan en el Herbario "Arturo E. Ragonese" (SF) de la FCAUNL) recolectados en el área analizada, producto de distintos trabajos realizados en el marco del Programa de Documentación, Conservación y valoración de la flora nativa que se desarrolla en la Facultad de Ciencias Agrarias (UNL).

Para comparar las distribuciones de las ecorregiones propuestas por Burkart et al. (1999), Brown y Pacheco (2006) y Morello et al. (2012), se accedió a los archivos digitales de la página de la Administración de Parques Nacionales (https://mapas.parquesnacionales.gob.ar/layers/geonode\%3Aarg ecorregiones_01_simpli), Argentina, Foro SCGISLatino (https://scgis-latino.blogspot.com) y archivo disponible en el CD de la publicación, respectivamente. 


\section{RESULTADOS}

Sumando todas las campañas, se recorrieron aproximadamente 7600 kilómetros de rutas nacionales, provinciales y caminos secundarios de acceso público, que se correspondieron con 2261 celdas hexagonales.

En terreno se registraron 1793 puntos de GPS de ubicación y asignación de bosques, los cuales vinculados al sistema de grillas se correspondieron con 1375 celdas de aproximadamente 541,63 ha cada una, representando un esfuerzo de muestreo cercano a las 750.000 ha. A estos datos de presencia de bosques se les sumaron 2201 hexágonos a partir del mapa provincial de OTBN.

En la Tabla 1 se detallan las especies arbóreas más relevantes observadas a campo y su asignación a cada clase de bosque nativo. Sobre la base de la información florística relevada a campo, de la bibliografía consultada y de las colecciones de herbario propias realizadas, en la Tabla 2 se presentan las especies leñosas más relevantes que permitieron caracterizar cada una de las ecorregiones presentes en el área analizada.

En la Figura 2 se observan las distribuciones estimadas para las clases de bosques de Quebrachales, Algarrobales, Bosques Mixtos y Bosques de Tres Quebrachos, derivadas de la aplicación del algoritmo del polígono envolvente y vinculadas al sistema de grillas hexagonales,

Clase Algarrobales (Fig. 2a): El 16.5\% de las celdas con datos de campo tuvieron presencia de bosques de esta clase, sin embargo, tuvo una amplia distribución en el área analizada. La distribución norte llega en el oeste hasta unos pocos kilómetros más al norte que la localidad de Pozo Borrado, mientras que en el este llega hasta la provincia de Chaco. El límite sur es el mismo que del área de estudio.
Clase Quebrachales (Fig. 2b): El 30,6 \% de las celdas con datos de campo tuvieron presencia de bosques de esta clase.

Clase Bosque Mixto (Fig. 2d): El 5.5 \% de las celdas con datos de campo tuvieron presencia de bosques de esta clase. Su distribución está en el noreste de la provincia de Santa Fe, teniendo su límite sur la localidad de Romang.

Clase Quebrachales de Tres Quebrachos (Fig. 2c): El 2\% de las celdas con datos de campo tuvieron presencia de bosques de esta clase. Su distribución está acotada al extremo noroeste de la provincia de Santa Fe, teniendo su límite sur la localidad de Villa Minetti.

Desde el punto de vista florístico, la ecorregión del Espinal no presenta especies leñosas (arbóreas y arbustivas) que sean exclusivas de ella, ya que la mayor parte de estas especies son compartidas con las ecorregiones chaqueñas. No ocurre lo mismo con las ecorregiones chaqueñas (Chaco Húmedo y Chaco Seco), en las que crecen numerosas especies exclusivas de ellas (Tabla 2). Por otra parte, varios elementos florísticos permiten a su vez separar claramente las ecorregiones de Chaco Húmedo y Chaco Seco (Tabla 2).

A partir del análisis de la distribución de remanentes de distintas clases de bosques, y de los análisis florísticos, se proponen nuevos límites para las ecorregiones del centro norte de la provincia de Santa Fe (Fig. 3). 


\section{J. F. Pensiero et al.}

Tabla 1. Especies arbóreas más relevantes observadas a campo y su asignación a cada clase de bosque nativo (A: Algarrobal; Q: Quebrachal; BM: Bosque mixto; 3Q: Quebrachal de Tres quebrachos). Table 1. Most relevant tree species observed in the field their assignment to each native forest class (A: Algarrobal; Q: Quebrachal; BM: Bosque mixto; 3Q: Quebrachal de Tres quebrachos).

\begin{tabular}{|c|c|c|c|c|}
\hline \multirow[t]{2}{*}{ Especies (Familias) } & \multicolumn{4}{|c|}{ Clase de bosque } \\
\hline & A & Q & BM & $3 Q$ \\
\hline Acanthosyris falcata Griseb. (Cervantesiaceae) & & $\mathrm{x}$ & $\mathrm{x}$ & $\mathrm{x}$ \\
\hline Achatocarpus praecox Griseb. (Achatocarpaceae) & $x$ & $x$ & $x$ & $x$ \\
\hline Aspidosperma quebracho-blanco Schltdl. (Apocynaceae) & $\mathrm{x}$ & $\mathrm{x}$ & $\mathrm{x}$ & $\mathrm{x}$ \\
\hline Aspidosperma triternatum Rojas Acosta (Apocynaceae) & & $\mathrm{x}$ & $\mathrm{x}$ & $\mathrm{x}$ \\
\hline Butia yatay (Mart.) Becc. (Arecaceae) & & & $\mathrm{x}$ & \\
\hline Celtis iguanaea (Jacq.) Sarg. (Cannabaceae) & & $\mathrm{x}$ & $\mathrm{x}$ & \\
\hline Celtis pallida Torr. (Cannabaceae) & $\mathrm{x}$ & $\mathrm{x}$ & $\mathrm{x}$ & $\mathrm{x}$ \\
\hline $\begin{array}{l}\text { Chloroleucon tenuiflorum (Benth.) Barneby \& J.W. Grimes } \\
\text { (Fabaceae) }\end{array}$ & & & $\mathrm{x}$ & \\
\hline Copernicia alba Morong (Arecaceae) & & $\mathrm{x}$ & & \\
\hline Cordia americana (L.) Gottschling \& J.S. Mill. (Boraginaceae) & & $\mathrm{x}$ & $\mathrm{x}$ & \\
\hline Cynophalla retusa (Griseb.) X. Cornejo \& H.H. Iltis (Capparaceae) & $\mathrm{x}$ & $\mathrm{x}$ & & \\
\hline Diplokeleba floribunda N.E. Br. (Sapindaceae) & & $\mathrm{x}$ & $\mathrm{x}$ & \\
\hline Ficus luschnathiana (Miq.) Miq. (Moraceae) & & $\mathrm{x}$ & $\mathrm{x}$ & \\
\hline Geoffroea decorticans (Gillies ex Hook. \& Arn.) Burkart (Fabaceae) & $\mathrm{x}$ & $\mathrm{x}$ & $\mathrm{x}$ & $\mathrm{x}$ \\
\hline Gleditsia amorphoides (Griseb.) Taub. (Fabaceae) & & $\mathrm{x}$ & $\mathrm{x}$ & \\
\hline Handroanthus heptaphyllus (Vell.) Mattos (Bignoniaceae) & & & $\mathrm{x}$ & \\
\hline Holocalyx balansae Micheli (Fabaceae) & & & $\mathrm{x}$ & \\
\hline Libidibia paraguariensis (D. Parodi) G.P. Lewis (Fabaceae) & & $\mathrm{x}$ & $\mathrm{x}$ & $\mathrm{x}$ \\
\hline Luehea divaricata Mart. (Tiliaceae) & & & $\mathrm{x}$ & \\
\hline $\begin{array}{l}\text { Maclura tinctorea (L.) Steud. ssp. mora (Griseb.) Vázq.Avila } \\
\text { (Moraceae) }\end{array}$ & & & $\mathrm{x}$ & \\
\hline Myracrodruon balansae (Engl.) Santin (Anacardiaceae) & & & $\mathrm{x}$ & \\
\hline Myrcianthes cisplatensis (Cambess.) O. Berg Phil. (Myrtaceae) & & $\mathrm{x}$ & $\mathrm{x}$ & \\
\hline Myrcianthes pungens (O. Berg) D. Legrand (Myrtaceae) & & & $\mathrm{x}$ & \\
\hline Myrsine laetevirens (Mez) Arechav. (Primulaceae) & & & $\mathrm{x}$ & \\
\hline Parkinsonia praecox (Ruiz \& Pav. ex Hook.) Hawkins (Fabaceae) & & & & $\mathrm{x}$ \\
\hline Phyllostylon rhamnoides (J. Poiss.) Taub. (Ulmaceae) & & & $\mathrm{x}$ & \\
\hline Prosopis affinis Spreng. (Fabaceae) & $\mathrm{x}$ & $\mathrm{x}$ & $\mathrm{x}$ & $\mathrm{x}$ \\
\hline Prosopis alba Griseb. (Fabaceae) & $\mathrm{x}$ & $\mathrm{x}$ & $\mathrm{x}$ & $\mathrm{x}$ \\
\hline Prosopis hassleri Harms var. nigroides Burkart (Fabaceae) & & $\mathrm{x}$ & & \\
\hline Prosopis kuntzei Harms (Fabaceae) & & & & $\mathrm{x}$ \\
\hline Prosopis nigra (Griseb.) Hieron. var. nigra (Fabaceae) & $\mathrm{x}$ & $\mathrm{x}$ & $\mathrm{x}$ & $\mathrm{x}$ \\
\hline Prosopis nigra (Griseb.) Hieron. var. ragonesei Burkart (Fabaceae) & & $\mathrm{x}$ & & \\
\hline Prosopis vinalillo Stuck. (Fabaceae) & $\mathrm{x}$ & & & \\
\hline Ruprechtia laxiflora Meisn. (Polygonaceae) & & $\mathrm{x}$ & $\mathrm{x}$ & $\mathrm{x}$ \\
\hline Sapindus saponaria L. (Sapindaceae) & & & $\mathrm{x}$ & \\
\hline Sarcomphalus mistol (Griseb.) Hauenschild (Rhamnaceae) & & $\mathrm{x}$ & & $\mathrm{x}$ \\
\hline Schinopsis balansae Engler (Anacardiaceae) & & $\mathrm{x}$ & $\mathrm{x}$ & $\mathrm{x}$ \\
\hline Schinopsis heterophylla Ragonese \& J. Castillo (Anacardiaceae) & & & & $\mathrm{x}$ \\
\hline Schinopsis lorentzii (Griseb.) Engl. (Anacardiaceae) & & & & $\mathrm{x}$ \\
\hline Senegalia praecox (Griseb.) Seigler \& Ebinger (Fabaceae) & & $\mathrm{x}$ & $\mathrm{x}$ & $\mathrm{x}$ \\
\hline Syagrus romanzoffiana (Cham.) Glassman (Arecaceae) & & & $\mathrm{x}$ & \\
\hline Tabebuia nodosa (Griseb.) Griseb. (Bignoniaceae) & & $\mathrm{x}$ & $\mathrm{x}$ & $\mathrm{x}$ \\
\hline Tabernaemontana catharinensis A. DC. (Apocynaceae) & & $\mathrm{x}$ & $\mathrm{x}$ & \\
\hline Terminalia triflora (Griseb.) Lillo (Combretaceae) & & $\mathrm{x}$ & $\mathrm{x}$ & \\
\hline $\begin{array}{l}\text { Vachellia aroma (Gillies ex Hook. \& Arn.) Seigler \& Ebinger } \\
\text { (Fabaceae) }\end{array}$ & $\mathrm{x}$ & $\mathrm{x}$ & $\mathrm{x}$ & $\mathrm{x}$ \\
\hline Vachellia caven (Molina) Seigler \& Ebinger (Fabaceae( & $\mathrm{x}$ & $\mathrm{x}$ & $\mathrm{x}$ & $\mathrm{x}$ \\
\hline Zanthoxylum fagara (L.) Sarg. (Rutaceae) & & $\mathrm{x}$ & $\mathrm{x}$ & $\mathrm{X}$ \\
\hline Zanthoxylum rhoifolium Lam. (Rutaceae) & & $\mathrm{x}$ & $\mathrm{x}$ & $\mathrm{X}$ \\
\hline
\end{tabular}


Tabla 2. Especies leñosas (arbóreas y arbustivas) más relevantes (ordenadas alfabéticamente por familias) presentes en cada una de las ecorregiones del área analizada (E: Espinal; $\mathrm{CH}$ : Chaco Húmedo; CS: Chaco Seco).

Table 2. Most relevant Woody (tree and shrub) species (alphabetically ordered by families) present in each ecoregions for the analyzed area.

\begin{tabular}{|c|c|c|c|}
\hline \multirow{2}{*}{ Especies (Familias) } & \multicolumn{3}{|c|}{ Ecorregión } \\
\hline & $\mathrm{E}$ & $\mathrm{CH}$ & CS \\
\hline Achatocarpus praecox Griseb. (Achatocarpaceae) & $\mathrm{x}$ & $\mathrm{X}$ & $\mathrm{x}$ \\
\hline Aspidosperma quebracho-blanco Schltdl. (Apocynaceae) & $\mathrm{x}$ & $\mathrm{X}$ & $\mathrm{x}$ \\
\hline Celtis pallida Torr. (Cannabaceae) & $\mathrm{x}$ & $\mathrm{X}$ & $\mathrm{x}$ \\
\hline Maytenus vitis-idaea Griseb. (Celastraceae) & $\mathrm{x}$ & $\mathrm{X}$ & $\mathrm{x}$ \\
\hline Jodina rhombifolia (Hook. \& Arn.) Reissek (Cervantesiaceae) & $\mathrm{x}$ & $\mathrm{X}$ & $\mathrm{x}$ \\
\hline Geoffroea decorticans (Gillies ex Hook. \& Arn.) Burkart (Fabaceae) & $\mathrm{x}$ & $\mathrm{X}$ & $\mathrm{x}$ \\
\hline Prosopis affinis Spreng. (Fabaceae) & $\mathrm{x}$ & $\mathrm{X}$ & $\mathrm{x}$ \\
\hline Prosopis alba Griseb. (Fabaceae) & $\mathrm{x}$ & $\mathrm{X}$ & $\mathrm{x}$ \\
\hline Prosopis nigra (Griseb.) Hieron. (Fabaceae) & $\mathrm{x}$ & $\mathrm{X}$ & $\mathrm{x}$ \\
\hline Vachellia aroma (Gillies ex Hook. \& Arn.) Seigler \& Ebinger (Fabaceae) & $\mathrm{x}$ & $\mathrm{X}$ & $\mathrm{x}$ \\
\hline Vachellia caven (Molina) Seigler \& Ebinger (Fabaceae( & $\mathrm{x}$ & $\mathrm{X}$ & $\mathrm{x}$ \\
\hline Schinopsis balansae Engler (Anacardiaceae) & & $\mathrm{X}$ & $\mathrm{x}$ \\
\hline Tabebuia nodosa (Griseb.) Griseb. (Bignoniaceae) & & $\mathrm{X}$ & $\mathrm{x}$ \\
\hline Anisocapparis speciosa (Griseb.) X. Cornejo \& H.H. Iltis (Capparaceae) & & $\mathrm{x}$ & $\mathrm{x}$ \\
\hline Capparicordis tweediana (Eichler) H.H. Iltis \& X. Cornejo (Capparaceae) & & $\mathrm{X}$ & $\mathrm{x}$ \\
\hline Cynophalla retusa (Griseb.) X. Cornejo \& H.H. Iltis (Capparaceae) & & $\mathrm{X}$ & $\mathrm{x}$ \\
\hline Acanthosyris falcata Griseb. (Cervantesiaceae) & & $\mathrm{X}$ & $\mathrm{x}$ \\
\hline Erythroxylum cuneifolium (Mart.) O.E. Schulz. (Erythroxylaceae) & & $\mathrm{X}$ & $\mathrm{x}$ \\
\hline Libidibia paraguariensis (D. Parodi) G.P. Lewis (Fabaceae) & & $\mathrm{X}$ & $\mathrm{x}$ \\
\hline Senegalia praecox (Griseb.) Seigler \& Ebinger (Fabaceae) & & $\mathrm{X}$ & $\mathrm{x}$ \\
\hline Ruprechtia laxiflora Meisn. (Polygonaceae) & & $\mathrm{X}$ & $\mathrm{x}$ \\
\hline Sarcomphalus mistol (Griseb.) Hauenschild (Rhamnaceae) & & $\mathrm{X}$ & $\mathrm{x}$ \\
\hline Zanthoxylum fagara (L.) Sarg. (Rutaceae) & & $\mathrm{X}$ & $\mathrm{x}$ \\
\hline Zanthoxylum rhoifolium Lam. (Rutaceae) & & $\mathrm{X}$ & $\mathrm{x}$ \\
\hline Myracrodruon balansae (Engl.) Santin (Anacardiaceae) & & $\mathrm{x}$ & \\
\hline Annona emarginata (Schltdl.) H. Rainer (Annonaceae) & & $\mathrm{X}$ & \\
\hline Butia yatay (Mart.) Becc. (Arecaceae) & & $\mathrm{X}$ & \\
\hline Copernicia alba Morong (Arecaceae) & & $\mathrm{X}$ & \\
\hline Syagrus romanzoffiana (Cham.) Glassman (Arecaceae) & & $\mathrm{X}$ & \\
\hline Aspidosperma triternatum Rojas Acosta (Apocynaceae) & & $\mathrm{x}$ & \\
\hline Tabernaemontana catharinensis A. DC. (Apocynaceae) & & $\mathrm{X}$ & \\
\hline Handroanthus heptaphyllus (Vell.) Mattos (Bignoniaceae) & & $\mathrm{X}$ & \\
\hline Cordia americana (L.) Gottschling \& J.S. Mill. (Boraginaceae) & & $\mathrm{X}$ & \\
\hline Celtis iguanaea (Jacq.) Sarg. (Cannabaceae) & & $\mathrm{X}$ & \\
\hline Schaefferia argentinensis Speg. (Celastraceae) & & $\mathrm{X}$ & \\
\hline Terminalia triflora (Griseb.) Lillo (Combretaceae) & & $\mathrm{X}$ & \\
\hline Chloroleucon tenuiflorum (Benth.) Barneby \& J.W. Grimes (Fabaceae) & & $\mathrm{X}$ & \\
\hline Gleditsia amorphoides (Griseb.) Taub. (Fabaceae) & & $\mathrm{X}$ & \\
\hline Holocalyx balansae Micheli (Fabaceae) & & $\mathrm{X}$ & \\
\hline Prosopis hassleri Harms var. nigroides Burkart (Fabaceae) & & $\mathrm{X}$ & \\
\hline Prosopis nigra (Griseb.) Hieron. var. ragonesei Burkart (Fabaceae) & & $\mathrm{X}$ & \\
\hline Prosopis vinalillo Stuck. (Fabaceae) & & $\mathrm{X}$ & \\
\hline Ficus luschnathiana (Miq.) Miq. (Moraceae) & & $\mathrm{X}$ & \\
\hline Maclura tinctorea (L.) Steud. ssp. mora (Griseb.) Vázq.Avila (Moraceae) & & $\mathrm{X}$ & \\
\hline Myrcianthes cisplatensis (Cambess.) O. Berg Phil. (Myrtaceae) & & $\mathrm{X}$ & \\
\hline Myrcianthes pungens (O. Berg) D. Legrand (Myrtaceae) & & $\mathrm{x}$ & \\
\hline Myrsine laetevirens (Mez) Arechav. (Primulaceae) & & $\mathrm{X}$ & \\
\hline Allophylus edulis (A. St-Hil., A. Juss. \& Cambess.) Hieron. ex Niederl. (Sapindaceae) & & $\mathrm{X}$ & \\
\hline Diplokeleba floribunda N.E. Br. (Sapindaceae) & & $\mathrm{X}$ & \\
\hline Sapindus saponaria L. (Sapindaceae) & & $\mathrm{X}$ & \\
\hline Luehea divaricata Mart. (Tiliaceae) & & $\mathrm{X}$ & \\
\hline Phyllostylon rhamnoides (J. Poiss.) Taub. (Ulmaceae) & & $\mathrm{X}$ & \\
\hline Schinopsis lorentzii (Griseb.) Engl. (Anacardiaceae) & & & $\mathrm{x}$ \\
\hline Schinopsis heterophylla Ragonese \& J. Castillo (Anacardiaceae) & & & $\mathrm{x}$ \\
\hline Atamisquea emarginata Miers ex Hook. \& Arn. (Capparaceae) & & & $\mathrm{x}$ \\
\hline Monteverdia spinosa (Griseb.) Biral (Celastraceae) & & & $\mathrm{x}$ \\
\hline Parkinsonia praecox (Ruiz \& Pav. ex Hook.) Hawkins (Fabaceae) & & & $\mathrm{x}$ \\
\hline Prosopis kuntzei Harms (Fabaceae) & & & $\mathrm{x}$ \\
\hline Prosopis reptans Benth. (Fabaceae) & & & $\mathrm{x}$ \\
\hline Prosopis ruscifolia Griseb. (Fabaceae) & & & $\mathrm{x}$ \\
\hline Prosopis sericantha Gillies ex Hook. \& Arn (Fabaceae) & & & $\mathrm{x}$ \\
\hline Porlieria microphylla (Baill.) Descole, O’ Donell \& Lourteig (Zygophyll & & & $\mathrm{x}$ \\
\hline
\end{tabular}

Revista FAVE - Ciencias Agrarias 20 (1) 2021 | 

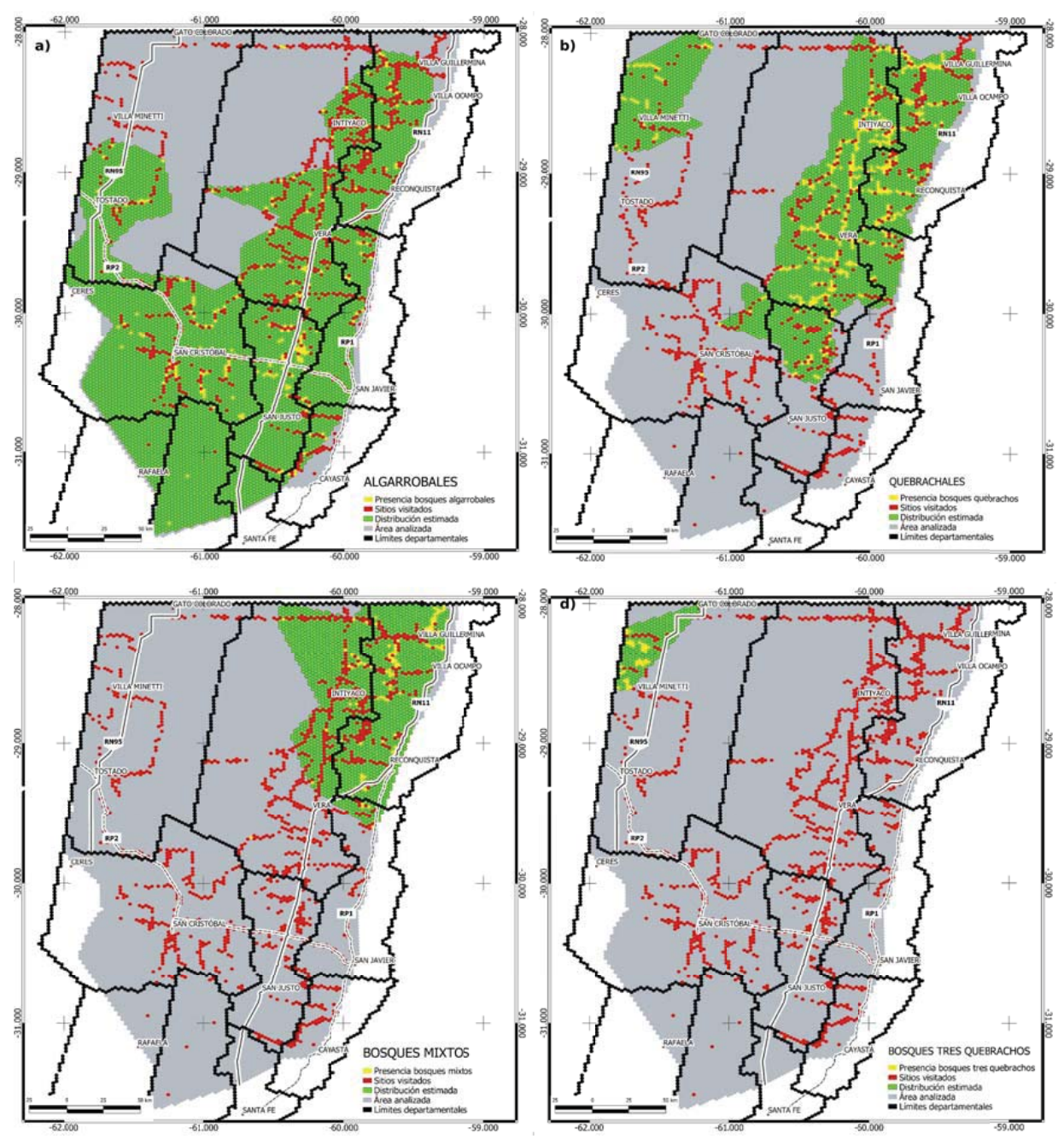

Figura 2. Distribuciones estimadas (celdas verdes) para las clases de bosques de a) Algarrobales, b) Quebrachales, c) Bosques Mixtos y d) Bosques Tres Quebrachos, derivadas de la aplicación del algoritmo del polígono envolvente. En gris se delimita el área analizada, las celdas en rojo indican sitios con registros de campo y las celdas en amarillo, la presencia de cada una de las clases de bosque.

Figure 2. Estimated distributions (Green cells) for forest classes a) Algarrobales, b) Quebrachales, c) Bosques Mixtos y d) Bosques Tres Quebrachos, derived from the application of the envelope polygon algorithm. In grey the analyzed area is delimited, the cells in red indicate sites with field records and the cells in yellow, the presence of each of the forest classes. 


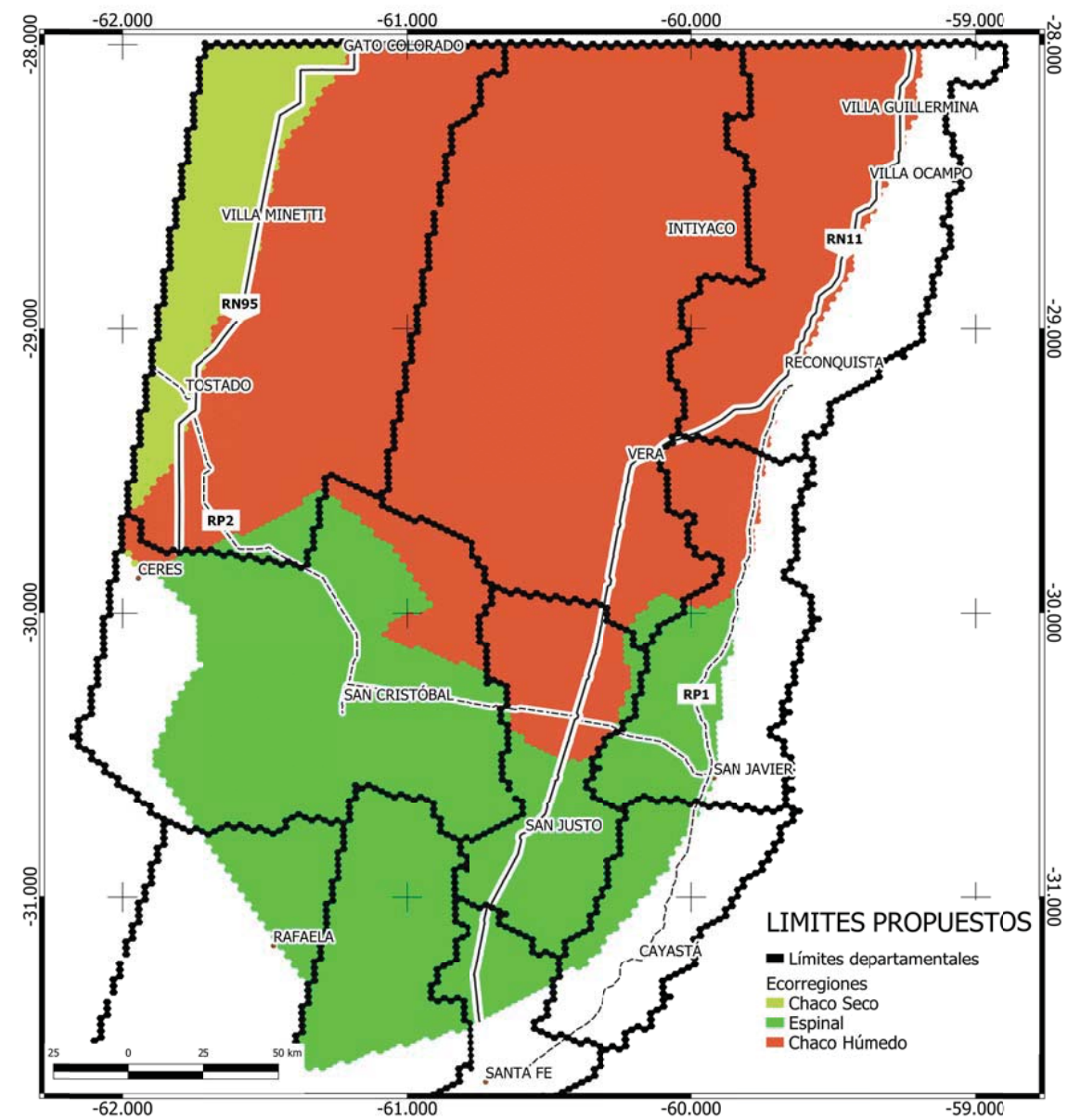

Figura 3. Límites que se han propuestos para las ecorregiones del norte de la Provincia de Santa Fe para el área analizada.

Figure 3. Proposed limits for the ecoregions of the north of Santa Fe province for the analyzed area. 


\section{DISCUSIÓN}

En esta propuesta sólo se analizó la distribución de límites de contacto de tres ecorregiones del centro-norte de Santa Fe: Chaco Seco, Chaco Húmedo y Espinal, por lo tanto no se discuten los límites de estos dos últimos con la ecorregión del Delta e Islas del Paraná. Cuando se la compara con los límites propuestos en la representación espacial digital de las Ecorregiones de Argentina, se observa que para el noroeste de la provincia, en el presente trabajo, el Chaco Seco abarca en forma continua toda el área que tiene como límite oeste el límite provincial con Santiago del Estero, su límite sur se ubica al norte de la localidad de Ceres, el límite este sigue a la ruta provincial 95 y su límite norte la localidad de Gato Colorado, limítrofe con la provincia de Chaco (Fig. 4a). En este sector se diferencia de las propuestas de Burkart et al. (1999) y de Morello et al. (2012), las cuales extienden el Chaco Húmedo hasta la esquina noroeste del límite con las provincias de Santiago del Estero y Chaco (Fig. 4b y 4d, respectivamente), y de Brown y Pacheco (2006), la cual restringe el ingreso del Chaco Seco en la provincia de Santa Fe a un área cercana al sur de la localidad de Tostado (Fig. 4c).

Hacia el centro y noreste de la provincia de Santa Fe (Fig. 5a), para el contacto de las ecorregiones del Espinal y del Chaco Húmedo, nuestra propuesta coincide en el límite sur del Chaco Húmedo con Burkart et al. (1999) y Morello et al. (2012) ubicándolo en cercanías de la localidad de Marcelino Escalada (Fig. 5b y 5d, respectivamente), a diferencia de Brown y Pacheco (2006), que lo llevan hasta la localidad de San Justo (Fig. 5 c). Desde el sur hacia el noreste, nuestra propuesta extiende el Espinal hasta casi la localidad de Alejandra (km 225 de la RP 1), mientras que las otras propuestas lo extienden hasta aproximadamente Colonia Teresa (km 180 de la RP 1). Y desde el sur hacia el noroeste, el borde de ambas ecorregiones acompaña el límite entre los departamentos San Cristóbal y Vera, coincidiendo bastante con Burkart et al. (1999), pero diferenciándose de las otras dos propuestas, que ubican ese límite más al sur.

La escala de ecorregiones es considerada como un primer marco para la toma de decisiones en lo referido a posibilidades de producción primaria basada en los bienes y recursos naturales, tales como los bosques nativos, pastizales, recursos fitogenéticos, etc. Pero también para las acciones de conservación y manejo de los remanentes de espacios naturales, a través de la aplicación de leyes como Ordenamiento Territorial de Bosques Nativos (Ley Provincial $N^{\circ}$ 13.372), Ley del Árbol (Ley Provincial $\left.\mathrm{N}^{\circ} 13.836\right)$ y acciones públicas: Ordenamiento territorial, Observatorio Santafesino de Suelos. En tal sentido, los límites propuestos en este trabajo pueden ser solicitados a los autores (en formato de archivo .tiff) a los fines pueda ser utilizado para ajustar los límites de las ecorregiones por medio de estudios complementarios de análisis de la vegetación (Torres et al., 2015) así como componentes de la fauna nativa (Fandiño y Giraudo, 2012). 


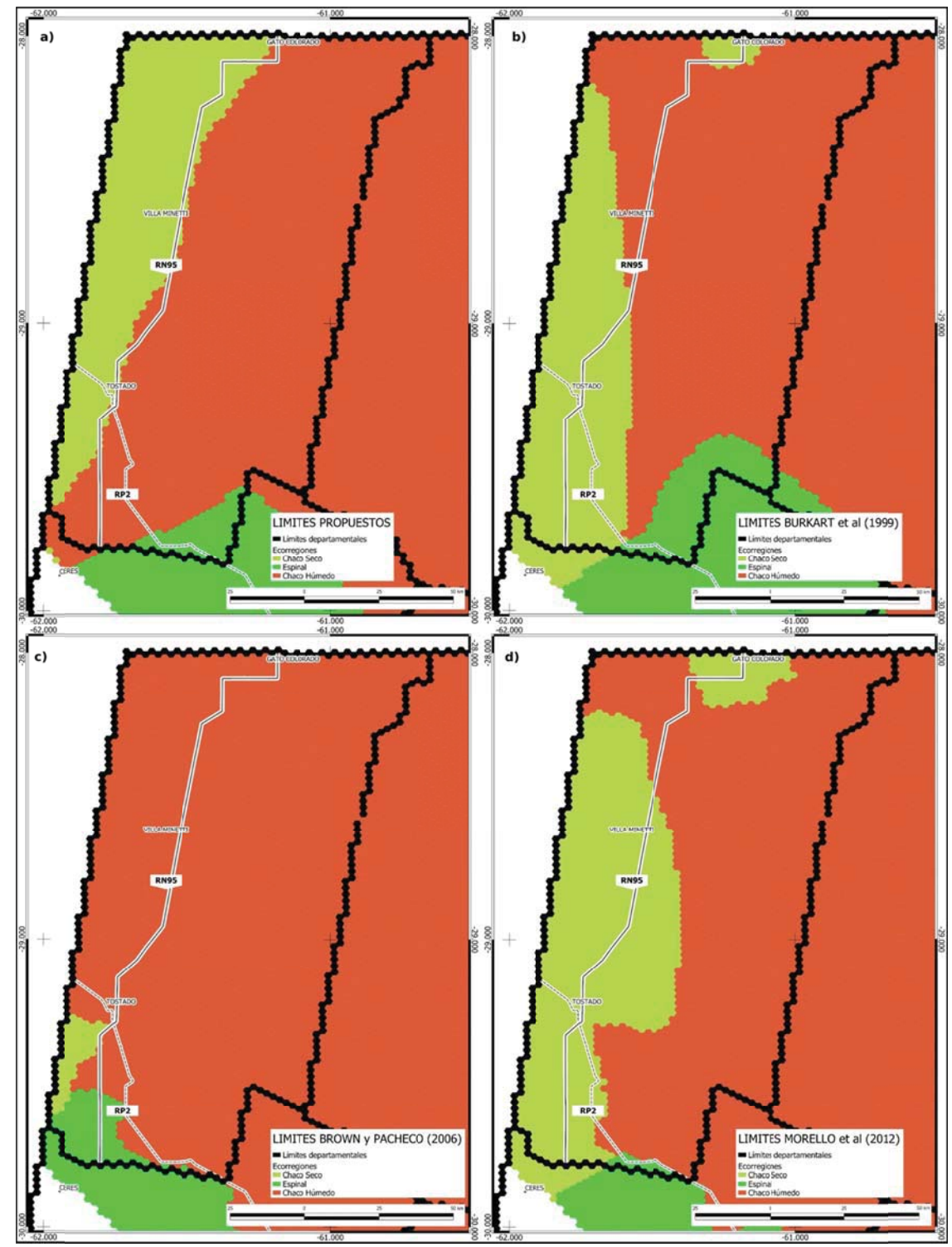

Figura 4. Distribución de las ecorregiones del Chaco Húmedo, Chaco Seco y Espinal para el noroeste de la provincia de Santa Fe según la propuesta a) del presente trabajo, b) de Burkart et al. (1999), c) de Brown y Pacheco (2006), y d) de Morello et al. (2012).

Figure 4. Distribution of Chaco Humid, Dry Chaco and Espinal ecoregions for the northwest of Santa Fe province according to: a) present work, b) Burkart et al. (1999), c) Brown y Pacheco (2006), and d) Morello et al. (2012). 

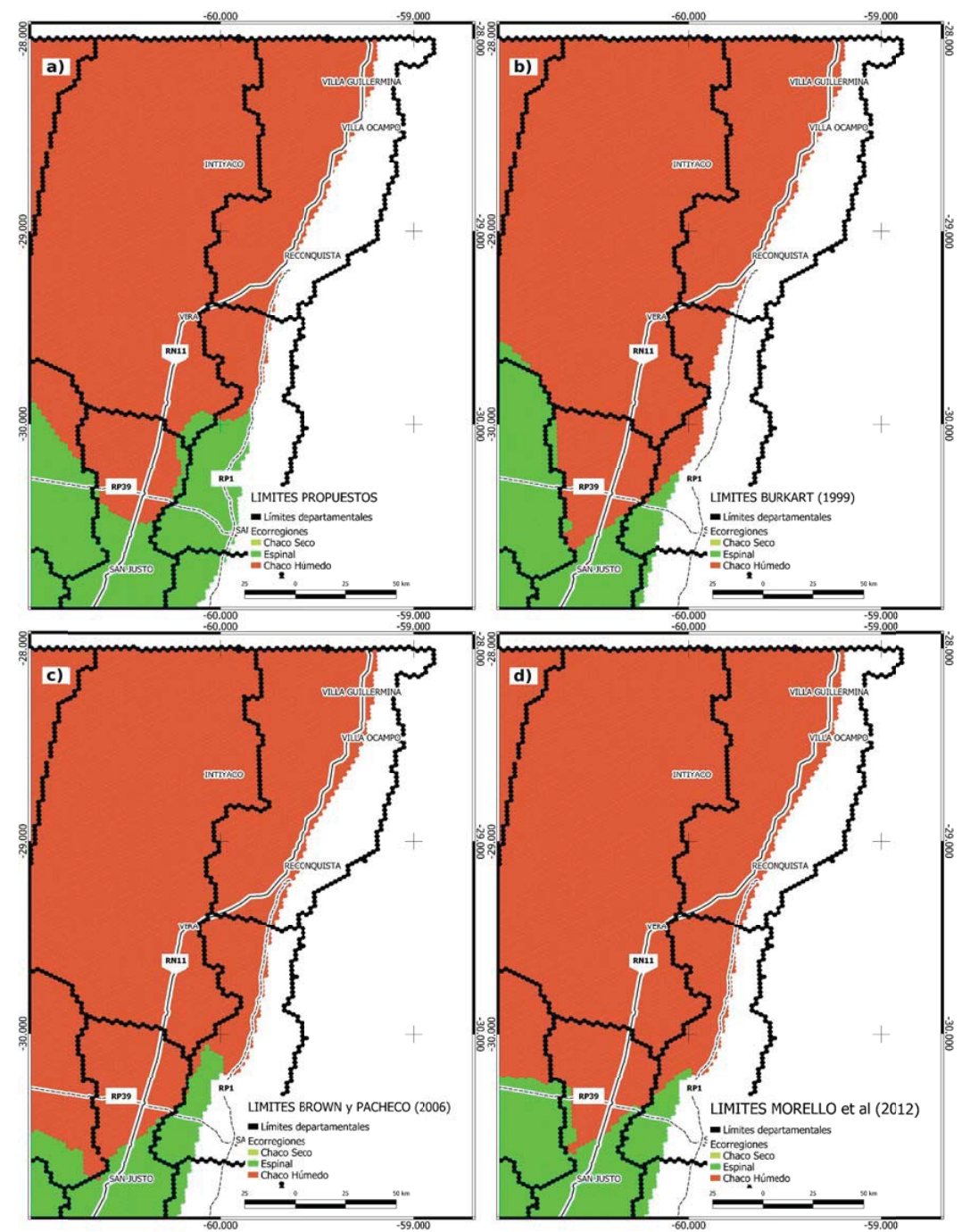

Figura 5. Distribución de las ecorregiones del Chaco Húmedo, Chaco Seco y Espinal para el noroeste de la provincia de Santa Fe según la propuesta a) del presente trabajo, b) de Burkart et al. (1999), c) de Brown y Pacheco (2006), y d) de Morello et al. (2012).

Figure 5. Distribution of Chaco Humid, Dry Chaco and Espinal ecoregions for the northeast of Santa Fe province according to: a) present work, b) Burkart et al. (1999), c) Brown y Pacheco (2006), and d) Morello et al. (2012). 


\section{BIBLIOGRAFÍA}

Barberis IM, Pire EF, Lewis JP. 1998. Spatian heterogeneity and woody species distribution in a Schinopsis balansae (Anacardiaceae) forest of the Southern Chaco, Argentina. Revista Biología Tropical 46(3): 515-524.

Biasatti NR. 2016. Las ecoregiones, su conservación y las Áreas Naturales Protegidas de la provincia de Santa Fe. Ministerio de Medio Ambiente. 244 pp.

Birch CP, Oom SP, Beecham J. 2007. Rectangular and hexagonal grids used for observation, experiment and simulation in ecology. Ecological Modelling. 206: 347-359.

Brown AD, Pacheco S. 2006. Propuesta de actualización del mapa ecorregional de la Argentina. En: Brown AD, Ortiz UM, Acerbi, M Corcuera J, editores. La situación ambiental argentina 2005. Fundación Vida Silvestre, Buenos Aires. Pp. 28-31

Burkart R, Bárbaro NO, Sánchez RO, Gómez DA. 1999. Ecorregiones de la Argentina, APN, PRODIA. 43 pp.

Cabrera AL. 1976. Regiones fitogeográfcas argentinas. En: Kugler WF editor. Enciclopedia Argentina de Agricultura y Jardinería. Tomo 2. 2da edición. Acme, Buenos Aires, Argentina. Fascículo 1. Pp. 1-85.

Carnevale NJ, Torres PS, Lewis JP. 1999. Análisis numérico y estacionalidad de las comunidades higrófilas de los Bajos Submeridionales santafesinos (Argentina). Boletín de la Sociedad Argentina de Botánica 34(1-2): 39-46.

D’Angelo C, Prado DE, Stofella SL, Lewis JP. 1987. The subchaquenian vegetation of province of Santa Fe (Argentina). Phytocoenologia 15(3): 329-352.

Fandiño B, Giraudo AR. 2012. Un análisis biogeográfico de la composición y distribución de la avifauna de Santa Fe, Argentina. Ornitología Neotropical 23(4): 467-488.
Hilgert NI, Pensiero JF, Marino G, Lewis JP, D'Angelo C. 2003. Vegetation of the Saladillo area (Province of Santa Fe) in the south of the Chaco Region, Argentina. Interciencia 28 (9): 512-520.

Lewis JP. 1981. La vegetación de la provincia de Santa Fe. Sociedad Argentina de Estudios Geográficos (GAEA) Serie Especial No 9: 121-148.

Lewis JP. 1991. Three levels of floristical variation in the forests of Chaco, Argentina. Journal of Vegetation Science 2: 125-130.

Lewis JP, Collantes MB. 1973. El Espinal periestépico. Ciencia e Invest. 29. 345-408.

Lewis JP, Pire EF. 1981. Reseña sobre la vegetación del Chaco Santafesino. INTA, Serie Fitogeográfica No 18. Pp 42.

Lewis JP, Pire EF, Prado DE, Stofella SL, Franceschi EA, Carnevale NJ. 1990. Plant communities and phytogeographical position of a large depression in the Great Chaco, Argentina. Vegetatio 86: 25-38.

Lewis JP, Pire EF, Vesprini JL. 1994. The mixed dense forest of the Southern Chaco. Contribution to the study of flora and vegetation of the Chaco. VIII. Candollea 49: 159-168.

Lewis JP, Pire EF, Barberis IM. 1997. Structure, physiognomy and floristic composition of a Schinopsis balansae (Anacardiaceae) forest in the Southern Chaco, Argentina. Revista Biología Tropical 49(3): 1013-1020.

Marino G, Pensiero JF. 2003. Heterogeneidad florística y estructural de los bosques de Schinopsis balansae (Anacardiaceae) en el chaco húmedo. Darwiniana 41 (1-4): 17-28.

Moreira A, Santos M. 2007. Concave hull: A k-nearest neighbours approach for the computation of the region occupied by a set of points. GRAPP. International Conference on Computer Graphics Theory and Applications. Barcelona, España. Pp. 61-68. 
Morello J, Rodriguez A. 2009. El Chaco sin bosques. Orientación Gráfica Editora S.R.L., Buenos Aires. 432 pp.

Morello J, Matteucci SD, Rodríguez AF, Silva M. 2012. Ecorregiones y complejos ecosistémicos argentinos. Orientación Gráfica Editora S.R.L., Buenos Aires. 752 pp. y un CD con mapas.

Oyarzabal M, Clavijo J, Oakley L, Biganzoli F, Tognetti P, Barberis I, León R J C. 2018. Unidades de vegetación de la Argentina. Ecología Austral. 28(1): 40-63.

Pensiero JF, Gutiérrez HF, Luchetti AM, Exner E, Kern V, Brnich E, Oakley L, Prado D, Lewis JP. 2005. Flora vascular de la provincia de Santa Fe. Claves para el reconocimiento de las familias y géneros. Catálogo sistemático de las especies. 403 pp.

QGIS Development Team. 2020. QGIS Geographic Information System. Open Source Geospatial Foundation Project. http://qgis. osgeo.org

Stoffella S. 1995. La heterogeneidad florística del pajonal de Spartina argentinensis (Poaceae) en los Bajos Submeridionales de la provincia de Santa Fe (Argentina). Boletín de la Sociedad Argentina de Botánica 31(12): 95-101.
Torrella S, Oakley L, Ginzburg R, Adámoli J, Galetto L. 2011. Estructura, composición y estado de conservación de la comunidad de plantas leñosas del bosque de tres quebrachos en el Chaco Subhúmedo Central. Ecología Austral 21: 179-188.

Torres Robles SS, Arturi MF, Contreras C, Peter G, Zeberio JM. 2015. Variaciones geográficas de la estructura y composición de la vegetación leñosa en el límite entre el Espinal y el Monte en el noreste de la Patagonia. Boletín de la Sociedad Argentina de Botánica 50:209-215.

Zarrilli AG. 2018. El bosque perdido. Una historia ambiental del norte santafesino (18902010). En: Brac M (Compiladora). El norte forestal: estudios sobre el territorio santafesino. Edición para el Ministerio de Gobierno y Reforma del Estado de la Provincia de Santa Fe. Consejo Federal de Inversiones. Pp 17-36. http://redaf.org.ar/version-digital-del-libro-el-norte-forestal-estudio-sobre-el-territorio-santafesino/ (Fecha de visita, 13/06/2020). 